\title{
THE INTEGRABILITY TENSOR FOR BUNDLE-LIKE FOLIATIONS
}

\author{
BY
}

RICHARD H. ESCOBALES, JR.

\begin{abstract}
A certain function is introduced which is useful in the study of a bundle-like foliation on a Riemannian manifold. Under the assumption that the leaves are totally geodesic, the Laplacian of this function is computed along a leaf. From this computation a sufficient condition is provided for the ambient manifold to be locally isometric to a product.
\end{abstract}

The principal result of this paper, Theorem 4.1, provides sufficient conditions for a compact foliated manifold which admits a bundle-like metric to be locally isometric to a product. The idea is to consider a function, $f$, defined on all of the ambient manifold and use the fact, that, under certain conditions, $f$ is subharmonic when regarded as a function along a leaf. In $\$ 1$ we recall the basic definitions needed in the remaining sections. $\$ 2$ contains a technical result, and $\$ 3$ gives the formula for the Laplacian of $f$ along a leaf.

Much of the work of $\S \S 1,2$ and 3 was done while the author held a Canisius College faculty fellowship in the summer of 1978. It was completed while he was on sabbatical in Syracuse. He wishes to thank Canisius College for both opportunities. He is grateful to Professor Jack Graver of Syracuse University for giving him faculty privileges while in Syracuse, to Professor Philip Church for the use of his office while he was away, to Glen Castore and to Professors Bruce Reinhart, Phil Parker, and Terry Bisson for their encouragement and advice, and to Professor Johnson who furnished a preprint [4].

1. The fundamental tensor. Let $M$ be a $C^{\infty}$ differentiable manifold, which throughout this paper is assumed to be connected and complete. Assume $M$ has a codimension $q$ foliation which is denoted by $\mathcal{V}$. Then this foliation may be defined by a maximal family of $C^{\infty}$ submersions $f_{\alpha}: U_{\alpha} \rightarrow R^{q}$ where $\left\{U_{\alpha}\right\}_{\alpha \in \Lambda}$ is an open cover of $M$, and where for each $p \in U_{\alpha} \cap U_{\beta}$ there is a local $C^{\infty}$ diffeomorphism, $\phi_{\beta \alpha}^{p}$, of $R^{q}$ so that $f_{\beta}=\phi_{\beta \alpha}^{p} \circ f_{\alpha}$ in some neighborhood $U_{p}$ of $p . U_{p}$ may be chosen so that it is in $U_{\alpha} \cap U_{\beta}$. In fact, if $p^{\prime} \in U_{\alpha} \cap U_{\beta}, \phi_{\beta \alpha}^{\beta}=\phi_{\beta \alpha}^{\beta^{\prime}}$ on $f\left(U_{p} \cap U_{p^{\prime}}\right)$ and $\phi_{\beta \alpha}^{p}=\phi_{\beta \gamma}^{p} \circ \phi_{\gamma \alpha}^{p}$ whenever this equation makes sense (see [6, pp. 2-3]). Observe that a tangent vector $V$ belongs to the tangent space of the distribution at $p, \mathcal{T}_{p}$, if and only if $f_{\alpha^{*} p} V=0$ or $V \in \operatorname{ker} f_{\boldsymbol{\alpha}^{*} p}$.

Received by the editors September 3, 1980 and, in revised form, February 23, 1981. Presented at the 780th meeting of the American Mathematical Society at Brown University, October 18, 1980.

1980 Mathematics Subiect Classification. Primary 57D30, 53C25. 
Now fix a Riemannian metric $\langle$,$\rangle on M$ and let $\nabla$ denote its Levi-Civita connection. Then the metric $\langle$,$\rangle determines an orthogonal distribution to \mathscr{V}$ which we denote by $\mathcal{H}$. Following [8] we define two tensors $T$ and $A$ on $M$ as follows:

$$
\begin{aligned}
& T_{E} F=\mathcal{V}_{\nabla \mho_{E}} \mathcal{H} F+\mathcal{H} \nabla_{\mho_{E}} \mathfrak{\mho} F, \\
& A_{E} F=\mathfrak{V} \nabla_{\mathscr{K} E} \mathcal{H} F+\mathscr{H} \nabla_{\mathcal{K} E} \mathfrak{V} F \text {. }
\end{aligned}
$$

Here $E$ and $F$ are arbitrary tangent vectors to $M$ and $\mathcal{V} E, \mathcal{H} E$, etc., are the projections onto the distributions $\mathscr{V}$ and $\mathcal{H}$.

The tensor $T$ is of type $(1,2)$ and enjoys the following properties [8, p. 460].

(a) $T_{E}$ is vertical; that is, $T_{E}=T_{\mho E}$.

(b) At each point, $T_{E}$ is a skew-symmetric linear operator on $M$ reversing the horizontal and vertical subspaces.

(c) For vertical vector fields $V$ and $W$ (i.e. vector fields tangent to the foliation $\mathfrak{V}$ ), $T_{V} W=T_{W} V$.

Likewise $A$ enjoys the following properties:

(a') $A_{E}$ is horizontal; $A_{E}=A_{\mathcal{H} E}$.

(b') At each point, $A_{E}$ is a skew-symmetric linear operator reversing the horizontal and vertical subspaces.

Definition 1.1. Let $M$ be foliated as above and suppose $\langle$,$\rangle is a Riemannian$ metric on $M .\langle$,$\rangle is called bundle-like if and only if for each \alpha, f_{\alpha}: U_{\alpha} \rightarrow R^{q}$ is a Riemannian submersion onto its image, or equivalently, the metric $\langle$,$\rangle on U_{\alpha}$ is projectible onto its image $f_{\alpha}\left(U_{\alpha}\right)$ in $R^{q}$ for each $\alpha$.

Notice, if $\langle$,$\rangle is bundle-like, the local diffeomorphisms \phi_{\beta \alpha}$ of $R^{q}$ are isometries with respect to the projected metrics. In general the metric projected onto $f_{\alpha}\left(U_{\alpha}\right)$ does not coincide with the flat metric.

Remark 1.2. The properties (a), (b), (c), (a') and ( $\left.\mathrm{b}^{\prime}\right)$ of the tensors $T$ and $A$ obtain whether or not the metric $\langle$,$\rangle is bundle-like on M$. (c) obtains because $T$ is essentially the second fundamental form for the leaf. The reader familiar with [8] knows that the tensor $A$ satisfies a third property called the alternating property on horizontal vectors:

(c') $\left(A_{X} Y\right)_{p}=-\left(A_{Y} X\right)_{p}$ for all $X, Y$ in $\mathcal{H}_{p}$ and for all $p \in M$. This property follows from the fact that $\langle$,$\rangle in [8] is bundle-like on M$. When $\langle$,$\rangle is bundle-like$ on $M, A$ is called the integrability tensor [2], since as O'Neill has shown [8, p. 461], $A_{X} Y=\frac{1}{2} \mathfrak{V}[X, Y]$ for any horizontal $X, Y$. The distribution $\mathcal{H}$ is integrable if and only if $A \equiv 0$ on $M$ (see [2, Corollary 1.5's proof]).

REMARK 1.3. A characterization of bundle-like metrics in terms of $\left(c^{\prime}\right)$ is given in [4, Lemma 1.2]. This result, communicated to me orally, was forgotten and rediscovered a year later. The Abstracts announcement [3, Theorem 1] did not take this conversation into account.

Now let us restrict ourselves to $U_{\alpha}$ and consider the submersions $f_{\alpha}: U_{\alpha} \rightarrow R^{q}$ which define the foliation $\mathfrak{V}$. We say a horizontal vector field $X$ on $U$ is $f_{\alpha}$ basic provided $f_{\alpha^{*} p} X=f_{\alpha^{*} p^{\prime}} X$ for every $p$ and $p^{\prime}$ in a connected component or plaque of $U_{\alpha} \cap L$ where $L$ is any leaf of $\mathcal{T}$. If $p$ and $p^{\prime}$ also lie on a plaque of $U_{\beta} \cap L$ with 
neighborhoods $U_{p}$ and $U_{p^{\prime}}$ of $p$ and $p^{\prime}$, respectively, contained in $U_{\alpha} \cap U_{\beta}$ and with $\phi \beta_{\beta \alpha}$ and $\phi \beta_{\alpha}^{\prime}$ defined on $f_{\alpha}\left(U_{p}\right)$ and $f_{\alpha}\left(U_{p^{\prime}}\right)$, respectively, then

$$
f_{\beta * p} X=\phi_{\beta \alpha^{*}}^{p} f_{\alpha^{*} p} X=\phi_{\beta \alpha^{*}}^{p^{\prime}} f_{\alpha^{*} p^{\prime}} X=f_{\beta * p^{\prime}} X
$$

whenever $p$ and $p^{\prime}$ both lie in $U_{p} \cap U_{p^{\prime}}$.

If $p$ and $p^{\prime}$ are not contained in $U_{p} \cap U_{p^{\prime}}$, we can choose a path in the plaque $U_{\alpha} \cap U_{\beta} \cap L$ connecting $p$ to $p^{\prime}$ and can select for each $x$ on that path an open $U_{x} \subset U_{\alpha} \cap U_{\beta}$ so $\phi_{\beta \alpha}^{x}$ is defined on $f_{\alpha}\left(U_{x}\right)$. By compactness of the path, every open cover has a finite subcover, so select one such finite subcover $\left\{U_{x_{i}}\right\}_{1 \leqslant i \leqslant n}$. If $\varepsilon$ is the Lebesgue number of the subcover, then choose $\left\{p_{k}\right\}_{0 \leqslant k \leqslant m}$ with $p_{0}=p, p_{m}=p^{\prime}$ so $d\left(p_{i}, p_{i+1}\right)<\varepsilon$, where $d$ is the distance function on the leaf induced from the metric on $\mathfrak{V}$. Then

$$
\phi_{\beta \alpha^{*}}^{p_{i *}} f_{\alpha_{i}} X=\phi_{\beta \alpha^{*}}^{i_{i+1}} f_{\alpha^{*} p_{i+1}} X
$$

so it follows that

$$
\begin{aligned}
& f_{\beta * p} X=f_{\beta * p_{0}} X=\phi_{\beta \alpha^{*}}^{P_{0}} f_{\alpha^{*} p_{0}} X=\phi_{\beta \alpha^{*}}^{P_{1}^{\prime}} f_{\alpha^{*} p_{1}} X \\
& =\cdots=\phi_{\beta_{\alpha^{*}}^{m}}^{m} f_{\alpha^{*} p_{m}} X=\phi_{\beta_{\alpha^{*}} p^{\prime}} f_{\alpha^{*} p^{\prime}} X=f_{\beta * p^{\prime}} X .
\end{aligned}
$$

We conclude $X$ is $f_{\beta}$ basic on $U_{\alpha} \cap U_{\beta}$ since we worked on an arbitrary $L$. Notice that we have not made any assumption that the metric $\langle$,$\rangle is bundle-like. We have$ established the following result.

Proposition 1.4. A horizontal vector field on $U_{\alpha} \cap U_{\beta}$ is $f_{\alpha}$ basic if and only if it is $f_{\beta}$ basic.

2. In this section we assume that $T \equiv 0$, that is, that the leaves of the foliation $\mathcal{V}$ are totally geodesic submanifolds of $M$. In addition, we will assume that the metric $\langle$,$\rangle is bundle-like on M$. We will show that if $X$ and $Y$ are $f_{\alpha}$ basic then $A_{X} Y=\frac{1}{2} \mathscr{V}[X, Y]$ satisfies the equation for a Killing vector field when restricted to a plaque of $U_{\alpha} \cap L$ : that is, we show

$$
\left\langle\nabla_{v}\left(A_{X} Y\right), W\right\rangle+\left\langle V, \nabla_{W}\left(A_{X} Y\right)\right\rangle=0
$$

for all $V, W$ tangent to the leaf on the given plaque. This result is more or less known [1], but our proof uses the equations of [8].

To begin, note

$$
\begin{aligned}
\left\langle\nabla_{V}\left(A_{X}, Y\right), W\right\rangle & =\left\langle\left(\nabla_{V} A\right)_{X} Y, W\right\rangle+\left\langle A_{\nabla_{V} X} Y, W\right\rangle+\left\langle A_{X} \nabla_{V} Y, W\right\rangle \\
& =\left\langle\left(\nabla_{V} A\right)_{X} Y, W\right\rangle+\left\langle A_{A_{X} V} Y, W\right\rangle+\left\langle A_{X} A_{Y} V, W\right\rangle .
\end{aligned}
$$

The second equality uses the definition of $A$, the fact that $V$ and $W$ are vertical, and that $\mathcal{H} \nabla_{V} X=A_{X} V$ for basic vector fields. Since $A$ is alternating on horizontal vector fields and $A_{\mathscr{C} E}$ is skew-symmetric, we get

$$
\left\langle\nabla_{V}\left(A_{X} Y\right), W\right\rangle=\left\langle\left(\nabla_{V} A\right)_{X} Y, W\right\rangle+\left\langle A_{X} V, A_{Y} W\right\rangle-\left\langle A_{Y} V, A_{X} W\right\rangle .
$$

In a similar way one sees

$$
\left\langle\nabla_{W}\left(A_{X} Y\right), V\right\rangle=\left\langle\left(\nabla_{W} A\right)_{X} Y, V\right\rangle+\left\langle A_{X} W, A_{Y} V\right\rangle-\left\langle A_{Y} W, A_{X} V\right\rangle .
$$


Adding the last two expressions we get

$$
\left\langle\nabla_{V}\left(A_{X} Y\right), W\right\rangle+\left\langle\nabla_{W}\left(A_{X} Y\right), V\right\rangle=\left\langle\left(\nabla_{V} A\right)_{X} Y, W\right\rangle+\left\langle\left(\nabla_{W} A\right)_{X} Y, V\right\rangle .
$$

Thus, to show $A_{X} Y$ satisfies Killing's equation on a plaque, it suffices to show

$$
\left\langle\left(\nabla_{V} A\right)_{X} Y, W\right\rangle+\left\langle\left(\nabla_{W} A\right)_{X} Y, V\right\rangle=0 .
$$

By one of the equations of [8],

$$
\left\langle R_{X V} Y, W\right\rangle=\left\langle\left(\nabla_{V} A\right)_{X} Y, W\right\rangle+\left\langle A_{X} V, A_{Y} W\right\rangle,
$$

since $T \equiv 0$. Using a Bianchi identity and (7) we get

$$
\left\langle R_{X V} Y, W\right\rangle=\left\langle R_{Y W} X, V\right\rangle=\left\langle\left(\nabla_{W} A\right)_{Y} X, V\right\rangle+\left\langle A_{Y} W, A_{X} V\right\rangle,
$$

or

$$
\left\langle R_{W Y} X, V\right\rangle=\left\langle\left(\nabla_{W} A\right)_{X} Y, V\right\rangle-\left\langle A_{Y} W, A_{X} V\right\rangle,
$$

since $\mathscr{V}\left(\nabla_{W} A\right)_{X} Y=-\mathcal{V}\left(\nabla_{W} A\right)_{Y} X$ by [8, p. 462]. Adding (7) and (9) and using a Bianchi identity, we obtain

$$
0=\left\langle\left(\nabla_{V} A\right)_{X} Y, W\right\rangle+\left\langle\left(\nabla_{W} A\right)_{X} Y, V\right\rangle .
$$

By (6) of this section $A_{X} Y$ is Killing on each plaque of $U_{\alpha} \cap L$. By Proposition 1.4 of the first section $X$ and $Y$ are $f_{\beta}$ basic on $U_{\alpha} \cap U_{\beta}$, so we have the following result.

Proposition 2.1. If $X$ and $Y$ are basic on $U_{\alpha}$ then $A_{X} Y$ is a Killing vector field on each plaque of $U_{\alpha} \cap L$ and each plaque of $U_{\alpha} \cap U_{\beta} \cap L$.

3. The function $f$. Throughout this and the next section the following conventions are observed:

(a) $1 \leqslant i, j, k, l \leqslant q$, where $q$ is the codimension of the foliation $X_{i}, X_{j}$, etc., are assumed horizontal.

(b) $1 \leqslant r \leqslant n-q$ where $n$ is the dimension of $M$. $V_{r}$ always denotes a vertical vector field.

(c) Whenever $\Sigma_{i, r}$ is used, we allow the indices to run through all of the permitted values.

In this section we study the function

$$
f(p)=\sum_{i, j}\left\langle A_{X_{i}} X_{j}, A_{X_{i}} X_{j}\right\rangle,
$$

where $\left\{X_{i}\right\}_{1 \leqslant i \leqslant q}$ is an orthonormal frame at $p$ for the horizontal space $\mathcal{H}_{p}$, and where, as before, $A$ denotes the integrability tensor. The same assumptions made in $\S 2$ obtain here: $\langle$,$\rangle is bundle-like and T \equiv 0$. We note that our definition of $f(p)$ does not depend on the choice of frame at $p$. If $\left\{Y_{k}\right\}$ is related to the frame $\left\{X_{i}\right\}$ by an orthogonal transformation of $\mathcal{H}_{p}$, then

$$
\sum_{k, l}\left\langle A_{Y_{k}} Y_{l}, A_{Y_{k}} Y_{l}\right\rangle=\sum_{i, j}\left\langle A_{X_{i}} X_{j}, A_{X_{i}} X_{j}\right\rangle .
$$

We omit this routine computation.

Our goal is to study the Laplacian of $f$ on a leaf, $\Delta_{\text {leaf }} f$, and to try to compute it in some reasonable way. Let $\left\{V_{r}\right\}_{1 \leqslant r \leqslant n-q}$ be a family of vertical vector fields 
orthonormal at $p$ satisfying $\left(\nabla_{V_{r}} V_{r}\right)(p)=0$. Then by Spivak [10, p. 194], for instance,

$$
\left(\Delta_{\text {leaf }} f\right)(p)=\left(\sum_{r} V_{r} V_{r} f\right)(p) .
$$

Likewise, we choose a family of horizontal $f_{\alpha}$ basic vector fields $\left\{X_{k}\right\}$, orthonormal at and near $p \in M$, which are $f_{\alpha^{*}}$ related to vector fields $\left\{X_{k}^{*}\right\}$ on $f_{\alpha}\left(U_{\alpha}\right)$ and where $1 \leqslant k \leqslant q$ in both cases. We have

$$
V_{r} f=\sum_{i, j} V_{r}\left\langle A_{X_{i}} X_{j}, A_{X_{i}} X_{j}\right\rangle=\sum_{i, j} 2\left\langle\nabla_{V_{r}}\left(A_{X_{i}} X_{j}\right), A_{X_{i}} X_{j}\right\rangle
$$

and

$$
\begin{aligned}
V_{r}^{2} f & =V_{r} V_{r} f \\
& =\sum_{i, j}\left\{2\left\langle\nabla_{V_{r}} \nabla_{V_{r}} A_{X_{i}} X_{j}, A_{X_{i}} X_{j}\right\rangle+2\left\langle\nabla_{V_{r}}\left(A_{X_{i}} X_{j}\right), \nabla_{V_{r}}\left(A_{X_{i}} X_{j}\right)\right\rangle\right\} \\
& =\sum_{i, j}\left\{2\left\langle\nabla_{V_{r}}\left(A_{X_{i}} X_{j}\right), \nabla_{V_{r}}\left(A_{X_{i}} X_{j}\right)\right\rangle-2\left\langle R_{V_{r}, A_{X_{i}} X_{j}} V_{r}, A_{X_{i}} X_{j}\right\rangle\right\} .
\end{aligned}
$$

In the last equality $R$ is the curvature tensor of $\nabla$. The equality obtains since $A_{X_{i}} X_{j}$ satisfies Killing's equations on each plaque of $U_{\alpha} \cap L$ and so the formulas of $[5, \mathrm{p}$. 56] apply. (Note in [5], Kobayashi considers $\frac{1}{2}\langle W, W\rangle$, where $W$ is a Killing vector field, and his curvature tensor differs from the above one by a sign.) It follows from the above computations that if we regard $f$ as a function along a single leaf, then the Laplacian of $f$ on the leaf, $\Delta_{\text {leaf }} f$, has the following expression at the point $p$ :

Proposition 3.1. Under the assumptions of this section the Laplacian of $f$ at $p$, $\left(\Delta_{\text {leaf }} f\right)(p)$, has the following expression with respect to the adapted system at $p$ :

$$
\begin{aligned}
\left(\Delta_{\text {leaf }} f\right)(p)= & \sum_{i, j, r} 2\left\langle\nabla_{V_{r}}\left(A_{X_{i}} X_{j}\right), \nabla_{V_{r}}\left(A_{X_{i}} X_{j}\right)\right\rangle \\
& -\sum_{i, j} 2 S_{\text {leaf }}\left(A_{X_{i}} X_{j}, A_{X_{i}} X_{j}\right),
\end{aligned}
$$

where $S_{\text {leaf }}$ is the Ricci tensor of the leaf.

Proof. The result follows immediately from the definition of the Ricci tensor.

REMARK 3.2. A nice formula for $(\Delta f)(p)$ where $\Delta f$ denotes the Laplacian of $f$ on $M$ can be computed. Results of this kind will be discussed elsewhere.

4. A geometric application. We are now in a position to state our main theorem as an application of the work of $\S \S 2$ and 3. As before, $\mathcal{H}=\mathfrak{V}^{\perp} \subset T M$.

THEOREM 4.1. Let $M$ be a compact connected Riemannian manifold with foliation $\mathfrak{V}$. Assume the metric on $M$ is bundle-like with respect to $\mathcal{V}$. If the leaves of $\mathcal{V}$ are totally geodesic and have quasi-negative Ricci curvature, then locally $M$ is isometric to a product of plaques of the foliations $\mathfrak{V}$ and $\mathcal{H}$. 
Proof. ${ }^{1}$ We follow the reasoning in [11]. Since $M$ is compact, $f(x)$ $=\Sigma_{i, j}\left\langle A_{X_{i}} X_{j}, A_{X_{i}} X_{j}\right\rangle$ attains a maximum at some $p \in M^{\prime}$ and so $f$ is bounded on each leaf of $\mathcal{V}$. Suppose $L$ is the leaf on which $f$ attains its maximum. Since $S_{\text {leaf }}(W, W) \leqslant 0$ for all vertical $W$, we have by Proposition 3.1 that $\Delta_{\text {leaf }} f \geqslant 0$, so $f$ is subharmonic on $L$. Recall the formula given in 3.1:

$$
\left(\Delta_{\text {leaf }} f\right)(x)=2\left\{\sum_{i, j, r}\left\langle\nabla_{V_{r}} A_{X_{i}} X_{j}, \nabla_{V_{r}} A_{X_{i}} X_{j}\right\rangle-\sum_{i, j} S_{\text {leaf }}\left(A_{X_{i}} X_{j}, A_{X_{i}} X_{j}\right)\right\} .
$$

The assumption that $f$ attains its maximum on $L$ means $f$ equals some constant $c$ on $L$. If $c>0$, then $f$ is nowhere zero on $L$. By assumption $S_{\text {leaf }}(W, W)<0$ at some point $q$ on $L$ for all nonzero $W$. On the other hand $\Delta_{\text {leaf }} f \equiv 0$ on $L$ since $f$ is constant. We have a contradiction unless $A_{X_{i}} X_{j}=0$ for all $i, j$ at $q$ so $f(q)=0$. Since $f$ is constant on $L, f(p)=0$. But $p$ was the point, where $f$ attained its maximum on $M$. We conclude $f \equiv 0$ on $M$. If $L$ is not orientable take the oriented double cover of $L$ and apply the same argument extending $f$ and $\Delta_{\text {leaf }} f$ in the obvious way.

Since $f \equiv 0$ on $M$, this means $A_{X_{i}} X_{j}=0$, or $A_{E}$ annihilates $\mathcal{H}$. Since $A_{E}$ is skew-symmetric reversing $\mathcal{H}$ and $\mathfrak{V}$, it follows $A_{E}$ annihilates $\mathfrak{V}$ so $A_{E} \equiv 0$ for all $E$ tangent to $M$. Now $\langle$,$\rangle on M$ is bundle-like so by Remark 2, $\$ 1$, $A_{X} Y=\mathfrak{T} \frac{1}{2}[X, Y]=0$ for any horizontal $X, Y$. This means $\mathcal{H}$ is integrable. By Proposition 2 of [9], a geodesic horizontal at one point is everywhere horizontal. It follows that the leaves of $\mathcal{H}$ are totally geodesic in $M$. By assumption, the leaves of $\mathfrak{V}$ are also totally geodesic.

To conclude the proof we note that since $\mathscr{V}$ and $\mathcal{H}$ are both integrable, locally $M$ is diffeomorphic to the product of a connected open set of a leaf of $\mathscr{V}$ and a connected open set of a leaf of $\mathcal{H}$. Call these open sets $F$ and $J$, respectively. We may choose $F$ and $J$ so that $F \times J \subset U_{\alpha}$ where $U_{\alpha}$ is one of the open sets of $\S 1$. Recalling that $f_{\alpha}$ : $U_{\alpha} \rightarrow f_{\alpha}\left(U_{\alpha}\right)$, we note that for each $z \in F, f_{\alpha}$, when restricted to $z \times J$, is bijective onto its image. Restricting $f_{\alpha}$ to $F \times J$ we have a Riemannian submersion onto its image with totally geodesic fibers and complementary distribution integrable $(T \equiv 0$ and $A \equiv 0$ ). These two conditions are precisely the conditions for the total space of a Riemannian submersion which has the structure of a fibered space to be a local Riemannian product by 6.4 and 6.5 of [7]. Since $F \times J$ is already diffeomorphic to a product we conclude it is isometric to a product. Hence, $M$ is locally isometric to $F \times J$ or $M$ is a local Riemannian product.

EXAMPLE 4.2. Consider $R^{3}$ with the standard axes. Choose a family of parallel planes each of which makes an angle $\alpha$ ( $\alpha$ irrational) with the $(x, y, 0)$ plane so that the plane passing through $(0,0,0)$ contains the $x$ axis. This construction gives a foliation of $R^{3}$ by the family of parallel planes. Now say $(x, y, z) \sim\left(x^{\prime}, y^{\prime}, z^{\prime}\right)$ if and only if $x-x^{\prime}, y-y^{\prime}$ and $z-z^{\prime}$ are all integers. The relation $\sim$ induces an equivalence on $R^{3}$. We observe that $(x, y, z)$ and $\left(x^{\prime}, y^{\prime}, z^{\prime}\right)$ both in the same plane of the foliation are equivalent if and only if $y=y^{\prime}, z=z^{\prime}$ and $x-x^{\prime}$ is an integer.

\footnotetext{
${ }^{1}$ We can relax the compactness condition on $M$ and obtain the same conclusions, provided we keep the other hypotheses and, in addition, require that each leaf of $\mathfrak{V}$ be compact.
} 
Put the following metric on $R^{3}$. The leaves of the foliation (the planes which cut the $x y$ plane and which are either parallel to the $x$ axis or contain it) are equipped with the hyperbolic metric of constant curvature -1 . Any line orthogonal to the plane of the foliation is given the usual Euclidean metric. Thus, we get a product metric on $R^{3}$. $\sim$ preserves the metric so $R^{3} \rightarrow R^{3} / \sim=T^{3}$ is an isometric covering. The images of the planes in $R^{3}$ described above are dense leaves in $T^{3}$, so we have a codimension one foliation of $T^{3}$. Take $M=S^{1} \times T^{3}$ where $S^{1}$ has the usual metric and $T^{3}$ has the metric given above. Each point $p \in M$ still passes through one and only one two-dimensional leaf. Obviously, the distribution complementary to the leaves is integrable and both distributions are totally geodesic since they arise from a local product structure [4, Proposition 1.3].

\section{REFERENCES}

1. R. L. Bishop, Clairaut submersions, Differential Geometry in Honor of K. Yano, Kinokuniya Bookstore Co., Tokyo, 1972, pp. 21-31.

2. Richard H. Escobales, Jr. Riemannian submersions with totally geodesic fibers, J. Differential Geom. 10 (1975), 253-276.

3. The integrability tensor for bundle-like foliations, Abstracts Amer. Math. Soc. 1 (1980), 526.

4. D. Johnson and L. Whitt, Totally geodesic foliations, J. Differential Geom. 15 (1980), 225-235.

5. S. Kobayashi, Transformation groups in differential geometry, Ergebnisse der Math., Band 93, Springer-Verlag, New York, 1972.

6. H. Blaine Lawson, Jr., The quantitative theory of foliations, CBMS Regional Conf. Ser. in Math., vol. 27, Amer. Math. Soc., Providence, R. I., 1977.

7. T. Nagano, On fibred Riemann manifolds, Sci. Papers College Gen. Ed. Univ. Tokyo 10 (1960), $17-27$.

8. B. O'Neill, The fundamental equations of a submersion, Michigan Math. J. 13 (1966), 459-469.

9. B. Rienhart, Foliated manifolds with bundle-like metrics, Ann. of Math. (2) 69 (1959), 119-131.

10. M. Spivak, A comprehensive introduction to differential geometry. 4, Publish or Perish, Boston, Mass., 1975.

11. $\mathrm{H}$. Wu, A remark on the Bochner technique in differential geometry, Proc. Amer. Math. Soc. 78 (1980), 403-408. 